\title{
Valores éticos de la protección radiológica en procedimientos de radiodiagnóstico e intervencionismo radiológico
}

\section{Ethical values of radiological protection in radiodiagnostic and radiological interventionism procedures}

\author{
Carlos Ubeda de la Cerda ${ }^{1 *}$, Pablo Soffia-Sánchez ${ }^{2}$, Alonso Inzulza-Contardo \\ Patricia Miranda-Gonzalez ${ }^{4}$, Gonzalo Aragón-Caqueo ${ }^{5}$ y Diego Aragón-Caqueo ${ }^{6}$ \\ ${ }^{1}$ Departamento de Tecnología Médica, Facultad de Ciencias de la Salud, Centro de Estudios en Ciencias Radiológicas, Universidad de Tarapacá, \\ Arica; ${ }^{2}$ Departamento de Imágenes, Facultad de Medicina, Clínica Alemana-Universidad del Desarrollo, Santiago; ${ }^{3}$ Servicio de Imagenología, Clínica \\ San José, Arica; ${ }^{4}$ Servicio de Hemodinamia, Hospital Luis Calvo Mackenna, Santiago; ${ }^{5}$ Escuela de Medicina, Pontificia Universidad Católica de \\ Chile, Santiago; ${ }^{6}$ Escuela de Medicina, Universidad de Valparaíso, Valparaíso. Chile
}

\section{Resumen}

Las aplicaciones médicas de las radiaciones ionizantes, y en particular los procedimientos de radiodiagnóstico e intervencionismo, son la principal fuente de radiación artificial a la población mundial. Las radiaciones ionizantes tienen el potencial de generar efectos adversos en la salud de las personas. Para hacer un uso más seguro y eficiente de estas radiaciones, la Comisión Internacional de Protección Radiológica y otras organizaciones internacionales han implementado un sistema de protección radiológica que se basa en tres pilares: justificación, optimización y límites. A su vez, estos pilares se sustentan en cuatro valores éticos fundamentales: beneficencia, prudencia, justicia y dignidad. Dado que la legislación chilena no tiene incorporados estos aspectos, ni tampoco hemos encontrado artículos científicos nacionales que aborden dichos temas para procedimientos de radiodiagnóstico e intervencionismo, el presente trabajo de revisión narrativa tiene como objetivo elaborar un documento de consulta para los profesionales de la salud que contenga de manera sucinta y con definiciones precisas los principales aspectos que hay que tener en cuenta para comprender cómo funciona el sistema de protección radiológica y sus valores éticos.

Palabras clave: Radiaciones ionizantes. Sistema de protección radiológica. Valores éticos.

\section{Abstract}

Medical applications of ionizing radiation and in particular diagnostic and interventional radiology procedures are the main source of artificial radiation to the world population. Ionizing radiation has the potential to generate adverse health effects in people. To make safer and more efficient use of these radiations, the International Commission on Radiological Protection together with other international organizations has implemented a system of radiological protection based on three pillars: justification, optimization, and limits. In turn, these pillars are based on four fundamental ethical values: beneficence, prudence, justice, and dignity. Given that Chilean legislation does not incorporate these aspects, nor have we found national scientific articles that address these issues for diagnostic and interventional radiology procedures, the present work of narrative review aims to develop a consultation document for health professionals, which contains in a concise manner and with precise definitions, key elements to understand how the radiological protection system works and its ethical values.

Key words: Ionizing radiation. Radiation protection system. Ethical values.

Correspondencia:

${ }^{*}$ Carlos Ubeda de la Cerda

E-mail: carlos.ubeda.uta@gmail.com
Disponible en internet: 03-12-2021

Rev Chil Radiol. 2021;27(4):164-169 www.resochradi.com 0717-9308 / @ 2021 Sociedad Chilena de Radiología. Publicado por Permanyer. Éste es un artículo open access bajo la licencia CC BY-NC-ND (https://creativecommons.org/licenses/by-nc-nd/4.0/). 


\section{Introducción}

Las aplicaciones médicas de las radiaciones ionizantes, y en particular los procedimientos de radiodiagnóstico (específicamente la tomografía computarizada) e intervencionismo, representan la principal fuente de radiación artificial a la población mundial ${ }^{1}$. También se consideran estos procedimientos radiológicos como situaciones de exposición planificada, que a su vez abarca categorías de exposición ocupacionales, exposiciones del público y exposiciones médicas de pacientes ${ }^{2}$.

Dado que las radiaciones ionizantes tienen el potencial de generar efectos nocivos para la salud de las personas $^{2}$, la comunidad internacional se ha venido organizado desde el año 1925, a través de la Comisión Internacional de Unidades y Medidas Radiológicas ${ }^{3}$, la Comisión Internacional de Protección Radiológica (ICRP) ${ }^{4}$, el Comité Científico de las Naciones Unidas sobre el Efecto de las Radiaciones Atómicas ${ }^{5}$ y la Agencia Internacional de Energía Atómica ${ }^{6}$, para hacer un uso más seguro y eficiente de estas radiaciones, proponiendo con tal objetivo un sistema de protección radiológica (SPR) como marco conceptual y práctico para dichos fines ${ }^{2}$.

El actual SPR y sus principios de justificación, optimización y limitación han evolucionado basándose en tres fuentes: la ciencia, los valores éticos y la experiencia acumulada en la práctica cotidiana de los profesionales de la protección radiológica. El SPR ha madurado sobre la necesidad de realizar un juicio de valor en la interpretación de los riesgos y su equilibrio con los beneficios que estas aplicaciones conllevan?.

Al revisar la normativa que regula el uso de las radiaciones ionizantes en Chile, vemos que no se tocan valores éticos al hablar y aplicar la protección radiológica en procedimientos de radiodiagnóstico e intervencionismo ${ }^{8,9}$. Existen algunos artículos nacionales que dan cuenta de ciertos aspectos éticos asociados a las aplicaciones médicas de las radiaciones ionizantes, pero ninguno se vincula con el SPR ${ }^{10-16}$. En el ámbito internacional existe un incipiente interés por avanzar en estos temas, siendo ejemplo de ello la publicación NN. 138 de la ICRP que identificó los valores éticos asociados con el SPR para exposiciones ocupacionales, públicas y médicas, y para la protección del medio ambiente ${ }^{7}$. Actualmente, el grupo de trabajo N. ${ }^{\circ} 109$ de la misma ICRP está elaborando un nuevo informe que presentará los aspectos éticos en el uso de la radiación en medicina, dirigido a la protección radiológica de los pacientes y destinado a profesionales médicos, pacientes, público y autoridades ${ }^{17}$.

Por todo lo anterior, el presente trabajo de revisión narrativa tuvo como objetivo ser un documento de consulta para los profesionales de la salud, que contenga de manera sucinta y con definiciones precisas los principales aspectos que hay que tener en cuenta para comprender cómo funciona el SPR, así como los valores éticos que lo fundamentan y ayudan a resolver los juicios de valor que se pueden presentar durante la ejecución de los procedimientos de radiodiagnóstico e intervencionismo, para cuidar la salud de los pacientes y del personal médico.

\section{Desarrollo}

\section{Efectos biológicos de las radiaciones ionizantes $^{2}$}

La mayoría de los efectos nocivos para la salud por exposición a radiaciones ionizantes pueden agruparse en dos categorías generales:

- Deterministas: son reacciones tisulares nocivas, debidas principalmente a la muerte 0 el defecto en el funcionamiento de las células tras recibir dosis elevadas de radiación. Su aparición se caracteriza por un umbral de dosis. Por encima de este, la gravedad de la lesión, incluyendo el deterioro de la capacidad de recuperación del tejido, aumenta.

- Estocásticos: son mutaciones de células somáticas o germinales. Las primeras se manifiestan como cáncer en los individuos expuestos, y las segundas se presentan como una enfermedad heredable en la progenie. Su aparición no está asociada a un umbral de dosis. En el caso del cáncer, los estudios epidemiológicos y experimentales proporcionan evidencia del riesgo de la radiación a dosis de alrededor de $100 \mathrm{mSv}$ o menores, aunque con incertidumbres. En el caso de las enfermedades heredables, aunque no existe evidencia directa de los riesgos de la radiación en las personas, las observaciones experimentales argumentan convincentemente que esos riesgos para las futuras generaciones deberían estar incluidos en el sistema de protección.

\section{Sistema de protección radiológica}

Como se indica en los principios fundamentales de seguridad $^{18}$, «el objetivo fundamental de la protección radiológica es proteger a las personas y el 
medio ambiente contra los efectos nocivos de las radiaciones ionizantes». Este objetivo debe alcanzarse sin limitar indebidamente la operación de instalaciones ni la realización de actividades que generan riesgos radiológicos. En consecuencia, el SPR tiene por objeto evaluar, gestionar y controlar la exposición a la radiación con el fin de reducir, en la medida en que sea razonablemente posible, los riesgos radiológicos, comprendidos tanto los riesgos de efectos en la salud como los riesgos para el medio ambiente.

El uso del modelo denominado «lineal sin umbral» es considerado actualmente el mejor enfoque práctico en la gestión del riesgo radiológico para la protección a dosis y tasas de dosis bajas, y es acorde con el «principio de prevención»"19.

Para cumplir sus objetivos, el SPR se fundamenta en tres principios:

- Justificación: cualquier decisión que altere la situación de exposición a radiación debería producir más beneficio que daño. Esto significa que toda decisión en virtud de la cual se introduzca una nueva fuente de radiación, se reduzca una exposición existente o se reduzca el riesgo potencial de exposición debería producir suficiente beneficio individual o social como para compensar el detrimento que causa dicha decisión. Se considera que el beneficio se aplica a los individuos, a la sociedad en su conjunto y también al medio ambiente.

- Optimización: la probabilidad de recibir exposiciones, la cantidad de personas expuestas y la magnitud de las dosis individuales deberían mantenerse tan bajas como sea razonablemente alcanzable, teniendo en cuenta factores económicos y sociales. Esto significa que el nivel de protección debería ser el mejor, en las circunstancias prevalecientes, maximizando el margen de beneficio en relación al daño. Debería haber restricciones en las dosis o en los riesgos de los individuos asociados a una fuente en particular con el fin de evitar resultados extremadamente injustos o no equitativos del procedimiento de optimización (restricciones de dosis o de riesgo, y niveles de referencia).

- Limitación: las exposiciones individuales no deben exceder los límites de dosis recomendados. Solo se aplica a situaciones de exposición planificada, que no sean la exposición médica de los pacientes o la exposición de biota no humana. Los límites de dosis reglamentarios los decide la autoridad reguladora teniendo en cuenta las recomendaciones internacionales, y se aplican a trabajadores e individuos en situaciones de exposición planificada.

\section{Valores éticos}

En 1974 se inició el debate sobre los principios de la bioética cuando el Congreso de los Estados Unidos de América creó la Comisión Nacional para la Protección de los Sujetos Humanos de Investigación Biomédica y del Comportamiento, para identificar los principios éticos básicos que deben regir la investigación con seres humanos en la medicina y las ciencias de la conducta. En 1978 se publicó el Informe Belmont ${ }^{20}$, en el que se distinguen tres principios éticos básicos, por este orden: respeto por las personas, beneficencia y justicia. Sin embargo, en 1979, Tom L. Beauchamp y James F. Childress, en su famoso libro Principios de ética biomédica ${ }^{21}$, reformularon estos principios para ser aplicados a la ética asistencial o la toma de decisiones éticas en medicina, distinguiendo los valores éticos de no maleficencia, beneficencia, autonomía y justicia.

En el caso de las aplicaciones médicas de las radiaciones ionizantes y del sustento en término de los valores éticos que se deben considerar dentro de los principios del SPR, de acuerdo con lo propuesto por la ICRP tenemos:

- Beneficencia y no maleficencia: si la beneficencia consiste en prevenir el daño, eliminar el daño o hacer el bien a otros, la no maleficencia consiste en no causar daño a otros. Mientras que la no maleficencia implica la ausencia de acción, la beneficencia incluye siempre la acción. Se distinguen dos tipos de beneficencia: la beneficencia positiva y la utilidad. La beneficencia positiva requiere la provisión de beneficios. La utilidad requiere un balance entre los beneficios y los daños. La beneficencia puede entenderse, de manera más general, como todo tipo de acción que tiene por finalidad el bien de otros.

- Prudencia: capacidad de pensar, ante ciertos acontecimientos 0 actividades, sobre los posibles riesgos que estos conllevan, y adecuar o modificar la conducta para no recibir o producir perjuicios innecesarios. Es la capacidad de tomar decisiones informadas y cuidadosas, consideradas sin el pleno conocimiento del alcance y las consecuencias de los actos. También es la capacidad de elegir y actuar sobre lo que está en nuestro poder hacer y no hacer. Originalmente, prudencia significaba «sabiduría práctica». Describe la cualidad de tener 
conocimiento, experiencia y buen juicio para tomar decisiones razonadas y actuar en consecuencia.

- Justicia: incluye la distribución de ventajas y desventajas entre grupos de personas (justicia distributiva), justicia en la compensación de pérdidas (justicia restitutiva) y justicia en las normas y los procedimientos en los procesos de toma de decisiones (justicia procesal). También se puede entender como "dar a cada uno lo suyo". Finalmente, puede ser interpretada como el tratamiento equitativo y apropiado a la luz de lo que es debido a una persona.

- Dignidad: es un atributo de la condición humana, la idea de que algo se debe a una persona porque es humana (valor inherente del ser humano por el solo hecho de serlo). Esto significa que todo individuo merece un respeto incondicional, independientemente de los atributos o circunstancias personales como la edad, el sexo, la salud, la discapacidad, la condición social, el origen étnico, la religión, etc. La autonomía personal es un corolario de la dignidad humana. Es la idea de que los individuos tienen la capacidad de actuar libremente (es decir, de tomar decisiones sin coerción y con conocimiento de causa $)^{7,22}$.

\section{Discusión}

Cuando se decide realizar un procedimiento de radiodiagnóstico o intervencionismo, sin lugar a duda entran en juego una serie de cuestionamientos éticos y juicios de valor. Primero, por parte del médico prescriptor, que define la necesidad de someter a un haz de rayos $X$ a su paciente. En segundo lugar, por el paciente, que simplemente confía o cuestiona, en el menor de los casos, exponerse a esta radiación ionizante. Tercero, por el equipo médico que ejecuta e informa el procedimiento, que debe hacer todo lo posible para la seguridad y la eficiencia del uso de los rayos $\mathrm{X}$, preocupándose no solo de la dosis recibida por el paciente y por ellos mismos, sino también por cuidar que las imágenes obtenidas tengan la calidad diagnóstica o terapéutica adecuada para alcanzar el objetivo médico. En esta etapa también se debería considerar la necesidad de hacer un seguimiento a los pacientes que, por ejemplo, superan cierto nivel de dosis que haga presumir la posterior aparición de efectos deterministas. También proponemos un cuarto nivel de cuestionamiento, en el que estarían todos los profesionales a cargo de la garantía y el control de calidad de los equipos.
Dada la naturaleza probabilística de los efectos estocásticos y las propiedades del modelo lineal sin umbral, resulta sumamente complejo establecer una distinción clara entre qué dosis de radiación es «segura» o «peligrosa», lo cual a su vez crea dificultades para buscar una explicación del control de los riesgos de la radiación. La principal implicación del modelo lineal sin umbral es que debe asumirse algún riesgo finito, aunque sea pequeño, y que el nivel de protección establecido está basado en lo que se estima aceptable. Sin embargo, la evidencia científica, junto con la experiencia acumulada, no aseguran una correcta interpretación de los principios básicos del SPR, dado que las situaciones específicas que se pueden dar durante los procedimientos de radiodiagnóstico e intervencionismo son dinámicas y únicas, y cada pacientes es diferente.

De acuerdo con Lochard ${ }^{23}$, la aplicación de los principios del SPR es un desarrollo permanente de juicios de valor que se deberían basar en los valores éticos fundamentales, es decir, hacer más bien que mal, evitar los riesgos innecesarios, establecer una distribución justa de las exposiciones y tratar a las personas con respeto.

Así, el principio de justificación se debería basar principalmente, aunque no en exclusiva, en el valor ético de beneficencia y no maleficencia, o más bien en que hacer el bien pese más que hacer el mal. Aquí lo que se debe perseguir es que el beneficio para las personas y la sociedad sea mayor que las desventajas asociadas en términos de riesgo de radiación. También este principio se asocia al valor ético de la prudencia, ya que parte del riesgo de radiación y su análisis está asociado con el uso del modelo lineal sin umbral.

En cuanto al principio de optimización, este utiliza las restricciones a las exposiciones individuales y los niveles de referencia para el diagnóstico con el fin de reducir las desigualdades en la distribución de las exposiciones entre los grupos expuestos. Esta es la piedra angular del sistema. Por un lado, es un principio de acción que permite la aplicación práctica de la prudencia, y por otro, permite la introducción de la equidad y la justicia en la distribución de las exposiciones entre los individuos expuestos, lo que hace referencia directa al valor ético de la justicia. En última instancia, teniendo en cuenta las circunstancias particulares en que las personas están expuestas, así como sus preocupaciones y expectativas, el principio de optimización es respetar a dichas personas y tratarlas con dignidad. 
En tercer lugar, el principio de limitación exige que todas las exposiciones individuales y no médicas no superen los límites de dosis recomendados. Al igual que el principio de optimización, se refiere directamente al valor ético de la prudencia, pero más aún a la justicia, al restringir el riesgo de manera equitativa para una determinada situación de exposición planificada y categoría de exposición.

Finalmente, el cambio más grande que ha experimentado la medicina durante el último siglo es el paso de un modelo paternalista, en el que el médico sabía «lo que era bueno» para el paciente, a un modelo en que el sujeto es autónomo en las decisiones que afectan su salud y su cuerpo. Así, basados en el principio de autonomía (no considerado en la propuesta de la ICRP), no debemos olvidar que son el paciente y su entorno quienes pueden en todo momento aceptar o rechazar un procedimiento médico. Este principio se enlaza fuertemente con el deber de informar de manera veraz sobre los riesgos y los beneficios de las radiaciones ionizantes (concepto del consentimiento informado ${ }^{15}$ ), lo cual es sumamente complejo, pues se trata de un terreno de incertidumbre y de falta de datos cuantitativos. Para resolver este dilema, los profesionales de la salud deben recurrir al principio de justificación, es decir, si el procedimiento radiológico está justificado, y entonces se puede comunicar al paciente que los beneficios superan ampliamente los eventuales riesgos y que la recomendación es someterse a dicho procedimiento.

\section{Conclusiones}

Para realizar juicios de valor sobre la protección radiológica, tomando las mejores decisiones antes, durante y después de los procedimientos de radiodiagnóstico e intervencionismo, se debe recurrir a los principios básicos de justificación y optimización, que son confrontados desde el prisma de los valores de la ética biomédica.

Se deben buscar niveles razonables de protección (principio de optimización) y niveles de exposición tolerables (principio de limitación), actuando siempre con prudencia (principio de justificación), es decir, procurando hacer más bien que mal (beneficencia o no maleficencia), evitando la exposición innecesaria (prudencia), buscando una distribución justa de la exposición (justicia) y tratando a las personas con respeto (dignidad), sin olvidar su autonomía con respecto a acciones que afecten su cuerpo y su salud.
En realidad, las consideraciones éticas al aplicar el SPR son más complejas, ya que también existe la posibilidad de que el beneficio para el paciente cause daños a otros, como el personal médico, que también recibe alguna dosis, o familiares y amigos que pueden recibir alguna dosis dependiendo del tipo de procedimiento, y que también podrían obtener un beneficio indirecto derivado del beneficio médico al paciente.

\section{Agradecimientos}

El investigador Carlos Ubeda agradece el apoyo de la Dirección de Investigación de la Universidad de Tarapacá, a través del proyecto de investigación UTA Mayor N. ${ }^{\circ} 7713-18$.

\section{Financiamiento}

No hubo ningún tipo de financiamiento.

\section{Conflicto de intereses}

No existe ningún tipo de conflictos de intereses por parte de los autores.

\section{Responsabilidades éticas}

Protección de personas y animales. Los autores declaran que para esta investigación no se han realizado experimentos en seres humanos ni en animales.

Confidencialidad de los datos. Los autores declaran que en este artículo no aparecen datos de pacientes.

Derecho a la privacidad y consentimiento informado. Los autores declaran que en este artículo no aparecen datos de pacientes.

\section{Bibliografía}

1. United Nations Scientific Committee on Effects of Atomic Radiations (UNSCEAR). Sources and effects of ionizing radiation. New York: United Nations; 2008.

2. International Commission on Radiation Protection (ICRP). The $2007 \mathrm{Re}-$ commendations of the International Commission on Radiological Protection. ICRP 103. Ann ICRP. 2007;37:2-4

3. Comisión Internacional de Unidades Radiológicas y Medidas (ICRU). (Acceso el 08/11/2020). Disponible en: www.icru.org.

4. Comisión Internacional de Protección Radiológica (ICRP). (Acceso el 08/11/2020). Disponible en: www.icrp.org.

5. Comité Científico de las Naciones Unidas sobre los Efectos de las Radiaciones Atómicas (UNSCEAR). (Acceso el 08/11/2020). Disponible en: www.unscear.org.

6. Agencia Internacional de Energía Atómica (IAEA). (Acceso el 08/11/2020). Disponible en: www.iaea.org. 
7. International Commission on Radiation Protection (ICRP). Ethical foundations of the system of radiological protection. ICRP 138. Ann ICRP. 2018;47:1-65.

8. Ministerio de Salud. Decreto Supremo N ․ㅡ 3. Reglamento de protección radiológica de instalaciones radiactivas. República de Chile, 3 de enero de 1985. (Acceso el 08/11/2020). Disponible en: https://www.bcn.cl/leychile/navegar?idNorma $=7282$.

9. Ministerio de Salud. Decreto Supremo N․ 133. Reglamento sobre autorizaciones para instalaciones radiactivas o equipos generadores de radiaciones ionizantes, personal que se desempeña en ellas, u opere tales equipos y otras actividades afines. República de Chile, 22 de mayo de 1984. (Acceso el 08/11/2020). Disponible en: https://www.bcn.cl/leychile/ navegar?idNorma $=9794$

10. Moënne K. Profesionalismo en el ejercicio de la radiología. Rev Chi Radiol. 2017;23:193-4.

11. Hevia J, Bosch E, Moënne K, García C. Deber ético del radiólogo: confidencialidad y secreto médico, respeto a la intimidad y dignidad de paciente. Rev Chil Radiol. 2018;24:2-4

12. García C. El principio de justicia en la práctica radiológica. Rev Chil Radiol. 2018;24:85-6.

13. García C, García C, Bosch E, Moënne K. Los conflictos de intereses en radiología. Consideraciones prácticas. Rev Chil Radiol. 2018;24:126-8.

14. García C. ¿Está el radiólogo expuesto a conflictos éticos en su práctica diaria? Rev Chil Radiol. 2017;23:46-7.
15. García C, Moënne K, Bosch E. Consentimiento informado en radiología. Rev Chil Radiol. 2018;24:45-7.

16. García C. La medicina defensiva en la práctica de la radiología. Rev Chil Radiol. 2019;25:2-4.

17. International Commission on Radiation Protection (ICRP). Task Group 109. Ethics in radiological protection for medical diagnosis and treatment. (Acceso el 08/11/2020). Disponible en: http://www.icrp.org/ icrp_group.asp?id=104.

18. Principios fundamentales de seguridad. Colección de Normas de Seguridad del OIEA N. ${ }^{\circ}$ SF-1. Viena: OIEA; 2007.

19. United Nations Educational, Scientific and Cultural Organization. The Precautionary Principle. Paris, France: UNESCO; 2005.

20. National Commission for the Protection of Human Subjects of Biomedical and Behavioral Research. The Belmont Report: Ethical Principles and Guidelines for the Protection of Human Subjects of Research. Bethesda, Md: The Commission; 1978.

21. Beauchamp T, Childress J. Principles of Biomedical Ethics. $4^{\text {th }}$ ed. New York/Oxford: Oxford University Press; 1994.

22. Siurana Aparisi JC. Los principios de la bioética y el surgimiento de una bioética intercultural. Veritas. 2010;(22):121-57.

23. Lochard J. First Thomas S. Tenforde topical lecture: The Ethics of Radiological Protection. Health Phys. 2016;110:201-10. 